Misha J. Davis-Gunter ${ }^{1}$

Hubert Löwenheim ${ }^{2}$

Kamakshi V. Gopal ${ }^{3}$

Ernest J. Moore

\section{The I' potential of the human auditory brainstem response to paired click stimuli}

${ }^{1}$ Auditory Neuroscience Laboratory, Department of Audiology and Speech Sciences, Michigan State University, East Lansing, MI, USA;

${ }^{2}$ Hoerforschungszentrum, HNI-Klinik

Tuebingen, Tuebingen, Germany;

${ }^{3}$ Department of Speech and Hearing Sciences, University of North Texas, Denton, TX; ${ }^{4}$ Department of Molecular Pharmacology and Biological Chemistry, Northwestern University Medical School, Chicago, IL, USA

\section{KEY WORDS:}

auditory brainstem responses, compound action potential, excitatory post-synaptic potential, generator potential, summating potential

RECEIVED/ACCEPTED:

November 10, 1999/March 22, 2000

\begin{abstract}
When stimulated with an appropriate stimulus, the hair cells of the organ of Corti depolarize, causing the release of a neurotransmitter substance, which excites afferent VIIIth nerve dendrites. It is reasonable to hypothesize that excitatory postsynaptic potentials (EPSPs) generated by the dendrites of the auditory nerve in turn initiate a compound action potential (CAP). The EPSP is thought to be the generator potential for the CAP, and may be recorded in auditory brainstem responses (ABRs) as the I' potential. Determining the anatomical origin of I' may enhance the sensitivity of the ABR technique in hair cell/dendrite/auditory nerve evaluations. Whether I' is of sensory or of neural origin is equivocal, and therefore $\mathrm{I}^{\prime}$ is not well understood. To investigate this dilemma, ABRs were recorded from human subjects using standard and paired-click stimuli, and using subtraction methods to generate a derived ABR. Two early peaks, designated as $\mathrm{I}^{\circ}$ and $\mathrm{I}^{\prime}$, occurred before wave $\mathrm{I}$ in the derived $\mathrm{ABR}$. It was hypothesized that peaks $\mathrm{I}^{\circ}$ and $\mathrm{I}^{\prime}$ represent the summating potential and the generator potential, generated by the cochlea and VIIIth nerve dendrites, respectively.
\end{abstract}

Scand Audiol 2001;30:50-60
ADDRESS FOR CORRESPONDENCE:

Ernest J. Moore, Department of

Molecular Pharmacology and

Biological Chemistry (S215),

Northwestern University Medical

School, 303 E. Chicago Ave., Chicago

IL 60611-3008, USA.

Tel: +1 3125031736 ,

fax: +13125031700 ,

e-mail: mooreer@northwestern.edu

\section{Introduction}

Several investigators have reported a potential recorded in the human auditory brainstem responses (ABRs), labeled as I' (Hughes \& Fino, 1980, 1985; Hughes et al., 1981; Moore et al., 1992), I (Benito et al., 1984), or BI (Moore \& Semela, 1989; Moore et al., 1992). The potential can be readily detected at high intensities using piezoelectric earphones (Hughes \& Fino, 1980, 1985; Hughes et al., 1981) or electrostatically shielded earphones (Moore et al., 1992), since stimulus artifact is at a minimum with these types of transducers. I' has a latency of less than a millisecond (Hughes \& Fino, 1980; Benito et al., 1984; Moore et al., 1992), and is easily recorded at high intensity levels. The results of the literature are not forthcoming as to a specific anatomical generator for I'.

Is I' derived from hair cells of the cochlea, and thus, a receptor potential, or is it a product of the distal afferent VIIIth nerve dendrites, indicative of excitatory postsynaptic potentials (EPSPs)? Of the two receptor potentials of the cochlea, the cochlear microphonic (CM) has been ruled out as the origin of $\mathrm{I}^{\prime}$, since $\mathrm{I}^{\prime}$ does not exhibit a phase reversal in response to clicks of opposite polarity (Hughes \& Fino, 1980; Moore et al., 
1992). Ruling out the possibility that $I^{\prime}$ might be the summating potential (SP) has been more problematic, however. It has been hypothesized that $\mathrm{I}^{\prime}$ represents summed EPSP activity originating in afferent dendrites of the auditory nerve (Moore et al., 1992). EPSPs have been recorded in the guinea pig (Palmer \& Russell, 1986; Siegel \& Dallos, 1986). Through pharmacological dissection, the summed activity of a residual 'EPSP-like' potential has been successfully separated from other gross cochlear potential components in the cat (Klinke et al., 1988) and in the guinea pig (Dolan et al., 1989). However, successful recordings of what is thought to be the EPSP in the human ABR have not yet been accomplished.

The compound action potential (CAP) is a gross potential recorded from the spiral ganglion neurons of the VIIIth nerve. The response is generated by the summation of underlying single-unit action potentials (AP) (Antoli-Candela \& Kiang, 1978). The AP is an 'all-or-none' response which exhibits electrophysiologic refractoriness to a preceding stimulus. Two refractory mechanisms are observed: the absolute refractory period and the relative refractory period. The absolute refractory period of a human auditory nerve AP was reported to be approximately $1.0 \mathrm{~ms}$ (Eggermont, 1985) to 1.2 ms (Özdamar \& Dallos, 1978). It is impossible for discharge activity of the auditory nerve to be 're-initiated' within the absolute refractory period. The relative refractory period is characterized by an exponentially decreasing threshold for the AP to a resting level with a time constant of 4-5 ms (Eggermont, 1985), making it difficult, but not impossible, for successive nerve discharges to be initiated.

The EPSP, however, is a graded potential, indicative that an increase in amplitude will occur as a result of an increase in the intensity of the stimulus (Furukawa, 1986). The EPSP is not characterized by refractory mechanisms in response to preceding stimuli, but is regulated by specific adaptation phenomena that develop in the transmitter release process (Furukawa et al., 1978), as well as in other basic mechanisms of synaptic transmission such as neurotransmitter re-uptake (Eggermont, 1985). Since the AP follows the graded potential, the EPSP, adaptation is also reflected in the AP (Eggermont \& Odenthal, 1974). As defined by Eggermont (1985), adaptation includes short-term as well as long-term auditory fatigue. He noted that the adaptation processes developed at a slower rate than the refractory mechanisms. Thus, both adaptation processes and refractory mechanisms should be thought of as individual processes, allowing the distinction of refractory-related alterations of the CAP from adaptation-related regulations of both the EPSP and the CAP.

Based on these electrophysiologic features, a stimulus that is transient in nature and presented within $1 \mathrm{~ms}$ of a preceding stimulus should fall within the absolute refractory period of the auditory nerve, and thus inhibit the generation of an AP. By making the first of the two stimuli a transient as well, short-term adaptation effects displayed in response to the second stimulus should be minimized. Unfortunately, the adaptation in the response cannot be ruled out completely. Furthermore, the amount of transmitter quanta remaining at the presynaptic site after the first stimulus is initiated - is directly related to the amplitude of the EPSP to the second stimulus. If the results of this stimulation pattern cause the complete depletion of transmitter quanta, the generation of an EPSP may not be possible. Still, it is hypothesized that the second of a paired-click stimulus, which is initiated within the time range of the absolute refractory period (viz., $1.0 \mathrm{~ms}$ ) of the first stimulus, will give rise to EPSP activity in afferent spiral ganglion dendrites. In a similar paradigm, Moore et al. (1992) reported the presence of evoked EPSPs without the presence of the CAP, thus allowing the summation of activity to be recorded as the generator potential in the human ABR. Therefore, the purpose of the present investigation was to use a paired click stimulus paradigm to successfully record the SP and to determine whether presumed summed EPSP activity is reflected in the ABR as the $\mathrm{I}^{\prime}$ potential.

\section{Methods and procedure}

Clicks of $0.1 \mathrm{~ms}$ duration were presented monaurally at $105 \mathrm{~dB}$ peSPL using an electrostatically shielded earphone (Madsen, $\mathrm{MSH}-$ 300) to the left ear of three normal hearing subjects. The subjects ranged in age from 17 to 23 years. Each subject was tested on four separate occasions and data were pooled. Individual subject data series encompassed the recording of one ABR using the standard-click 
stimulus followed by the recording of seven ABRs with the paired-click stimulus using the delta ' $\mathrm{t}$ ' intervals of $4.0 \mathrm{~ms}, 2.0 \mathrm{~ms}, 1.0 \mathrm{~ms}, 0.8$ $\mathrm{ms}, 0.4 \mathrm{~ms}, 0.2 \mathrm{~ms}$ and $0.1 \mathrm{~ms}$. The electronic subtraction of the standard-click ABR from the paired-click ABR yielded seven derived responses.

Modular components (Modular Instruments, Inc. $100 / 200$ series $-\mathrm{MI}^{2}$ ) were used to digitally generate the single-click and paired-click stimuli of alternating polarity. A custom-written software program implemented on a microcomputer (IBM series) initiated the stimuli and controlled the data collection phase. Clicks were routed to the earphone from a dual-function generator $\left(\mathrm{MI}^{2}, 208\right)$ to a dual attenuator $\left(\mathrm{MI}^{2}, 108\right)$, lowpass filter (Frequency Devices, 901F) set to a cutoff of $5 \mathrm{kHz}$ to obviate high frequency side lobes, and an amplifier (Technics, SE-9060). A data controller timer $\left(\mathrm{MI}^{2}, 214\right)$ created the simultaneous initiation of the electronic averager with the onset of the stimulus.

A gold-cup electrode (Grass) filled with a conducting gel (Medi-trace) was applied at the vertex ( $\mathrm{Cz}$; active), a clip-on electrode was attached to the ipsilateral earlobe (A1; reference), and another gold-cup electrode was applied to the forehead (Fpz; patient ground).
Inter-electrode impedance was kept at or below $1.0 \mathrm{kOhms}$, and was measured by a batteryoperated impedance meter (Grass, EZM5A). The electrodes routed the electrophysiologic activity to the AC coupled preamplifier and filter (Data, Inc., 2124), set to a bandwidth of $1 \times 10^{2}$ $-3 \times 10^{3} \mathrm{~Hz}$ ( $-3 \mathrm{~dB}$ points). The amplified electrophysiologic activity $\left(1.8 \times 10^{5}\right)$ was averaged a total of 2,048 times with a sweep time of $10 \mathrm{~ms}$, a dwell time of $10 \mu \mathrm{s}$, digitized at 12 bits, and quantized $\left(1 \times 10^{3}\right.$ data points), using the microcomputer-based system.

The amplitude and latency of the potentials were measured using the digital read-out of two interactive cursors. Response latency was measured from the onset of the electrical click at the earphone to the most prominent peak of $\mathrm{I}^{\circ}$, $\mathrm{I}^{\prime}$ and I. Wave V was noted for its peak amplitude and peak latency to imply normalcy of the data. Peak-to-peak amplitude was measured from the first positive peak to the next negative trough of the respective peak.

\section{The paired-click paradigm}

The paired-click stimulus paradigm involved the manipulation of ABRs that were elicited with the 'standard click' and ABRs elicited with a 'paired
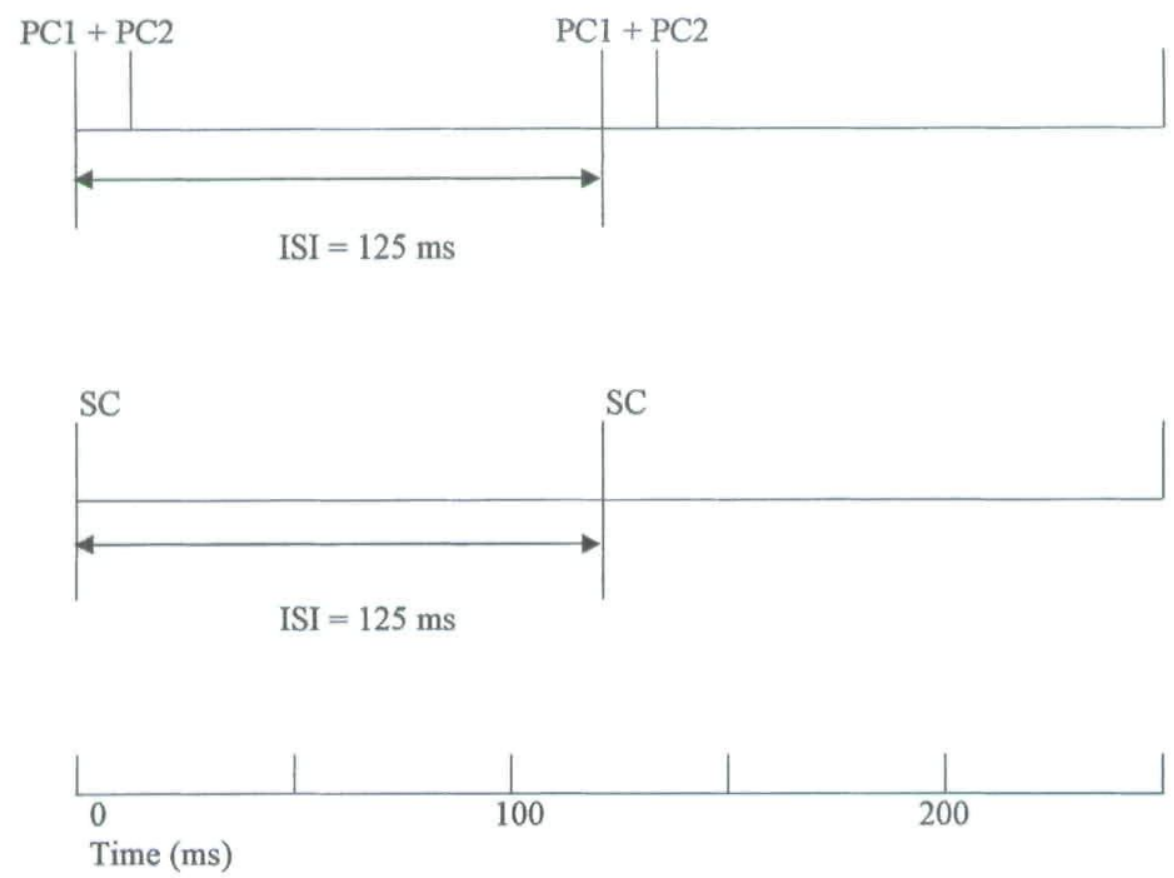

Fig. 1. Components of the paired-click and standard click stimulus paradigms. PC1, paired click 1; PC2, paired click 2; ISI, inter-stimulus interval; SC, standard click. 
Table I. Subtraction pairs and corresponding derived responses

\begin{tabular}{lll}
\hline Delta $t(\mathrm{~ms})$ & Subtraction pairs & Derived responses \\
\hline 4 & $4.0 \mathrm{t} \mathrm{ABR}-\mathrm{SC} \mathrm{ABR}$ & $\mathrm{D} / 4.0$ \\
2 & $2.0 \mathrm{t} \mathrm{ABR}-\mathrm{SC} \mathrm{ABR}$ & $\mathrm{D} / 2.0$ \\
1 & $1.0 \mathrm{t} \mathrm{ABR}-\mathrm{SC} \mathrm{ABR}$ & $\mathrm{D} / 1.0$ \\
0.8 & $0.8 \mathrm{t} \mathrm{ABR}-\mathrm{SC} \mathrm{ABR}$ & $\mathrm{D} / 0.8$ \\
0.4 & $0.4 \mathrm{t} \mathrm{ABR}-\mathrm{SC} \mathrm{ABR}$ & $\mathrm{D} / 0.4$ \\
0.2 & $0.2 \mathrm{t} \mathrm{ABR}-\mathrm{SC} \mathrm{ABR}$ & $\mathrm{D} / 0.2$ \\
0.1 & $0.1 \mathrm{t} \mathrm{ABR}-\mathrm{SC} \mathrm{ABR}$ & $\mathrm{D} / 0.1$ \\
\hline
\end{tabular}

click'. Fig. 1 illustrates the specific time intervals of the single-click and paired-click stimuli. The composition of the standard-click stimuli (SC) is simple relative to structural components; however, it is important to note that the pairedclick stimulus (PC) is comprised of two separate clicks: 'paired-click 1' (PC1) and 'paired-click 2' (PC2). The components of the paired-click stimulus, $\mathrm{PC} 1$ and $\mathrm{PC} 2$, are characterized by time intervals of $4.0 \mathrm{~ms}, 2.0 \mathrm{~ms}, 1.0 \mathrm{~ms}, 0.8 \mathrm{~ms}$, $0.4 \mathrm{~ms}, 0.2 \mathrm{~ms}$ and $0.1 \mathrm{~ms}$. These time intervals were chosen to be shorter than, encompass, as well as exceed the duration of the absolute (1.0 $\mathrm{ms})$ and relative (4-5 ms) refractory periods of the VIIIth nerve (Eggermont \& Odenthal, 1974). Varying the time intervals in separate ABR recording conditions resulted in seven different paired-click ABR responses.

The paired-click stimulus paradigm is based on specific stimulus response characteristics, as each of the paired-click components causes separate electrophysiologic effects. PC1 will have the effect of initiating action potentials (APs) along the auditory nerve, resulting in waves I', and waves I through V in the ABR. On the other hand, PC2 is delivered to the auditory system during times when additional APs should not be re-initiated. Although PC2 theoretically should not initiate an AP, it should initiate EPSP responses whose summed activity would be recorded in the ABR. The paired-click ABR includes the effects of PC1 and PC2 that make the identification of individual electrophysiologic effects impossible. In order to remove the effects of PC1, so that only PC2 effects remain, derived responses were obtained. These were calculated by subtracting the standard-click ABRs - whose electrophysiologic effects are identical to PC1 of the paired-click stimuli from individual paired-click ABRs. Table I illustrates the subtraction pairs and corresponding derived responses. Derived responses thus represent only those electrophysiologic effects of PC2, thus, revealing the presumed EPSP for the appropriate delta $t$ condition. In order to arrive at an accurate derived response, measures were taken to ensure that the SC of the standardclick stimulus $\mathrm{ABR}$ and $\mathrm{PC} 1$ of the paired-click stimulus ABR were identical. Thus, interstimulus intervals were kept at $125 \mathrm{~ms}$ (repetition rate $=8 / \mathrm{s}$ ) for both the standard-click and pairedclick recording conditions in order to minimize adaptation effects (Eggermont \& Odenthal, 1974). Table II illustrates specific interstimulus intervals for SC and PC measurements. It is important to note, however, that, theoretically, increased latency and decreased amplitude effects are not expected for an ISI $>128 \mathrm{~ms}$ (Eggermont \& Odenthal, 1974).

\section{Results}

The latencies and amplitudes of the three subjects for wave I (whose anatomical generator

Table II. Inter-stimulus intervals for standard-click and paired-click measurements

\begin{tabular}{|c|c|c|c|}
\hline Condition & ISI & Minimum ISI & Maximum ISI \\
\hline SC ABR & $\mathrm{SC}-\mathrm{SC}: 125 \mathrm{~ms}$ & $125 \mathrm{~ms}$ & $125 \mathrm{~ms}$ \\
\hline 4.0 tABR & $\mathrm{PC} 1$ - PC1: $125 \mathrm{~ms}$ & $\mathrm{PC} 1-\mathrm{PC} 2: 4.0 \mathrm{~ms}$ & $\mathrm{PC} 2$ - PC1: $121.0 \mathrm{~ms}$ \\
\hline $2.0 \mathrm{t} \mathrm{ABR}$ & $\mathrm{PC} 1$ - PC1: $125 \mathrm{~ms}$ & $\mathrm{PC} 1-\mathrm{PC} 2: 2.0 \mathrm{~ms}$ & $\mathrm{PC} 2$ - PC1: $123.0 \mathrm{~ms}$ \\
\hline $1.0 \mathrm{t} \mathrm{ABR}$ & PC1 - PC1: $125 \mathrm{~ms}$ & $\mathrm{PC} 1$ - PC2: $1.0 \mathrm{~ms}$ & $\mathrm{PC} 2$ - $\mathrm{PC} 1: 124.0 \mathrm{~ms}$ \\
\hline $0.8 \mathrm{t}$ ABR & $\mathrm{PC} 1$ - PC1: $125 \mathrm{~ms}$ & $\mathrm{PC} 1-\mathrm{PC} 2: 0.8 \mathrm{~ms}$ & $\mathrm{PC} 2$ - PC1: $124.2 \mathrm{~ms}$ \\
\hline $0.4 \mathrm{t} \mathrm{ABR}$ & $\mathrm{PC} 1$ - PC1: $125 \mathrm{~ms}$ & PC1 - PC2: $0.4 \mathrm{~ms}$ & $\mathrm{PC} 2$ - PC1: $124.6 \mathrm{~ms}$ \\
\hline $0.2 \mathrm{t} \mathrm{ABR}$ & $\mathrm{PC} 1$ - PC1: $125 \mathrm{~ms}$ & $\mathrm{PC} 1$ - PC2: $0.2 \mathrm{~ms}$ & $\mathrm{PC} 2$ - PC1: $124.8 \mathrm{~ms}$ \\
\hline $0.1 \mathrm{t} \mathrm{ABR}$ & PC1 - PC1: $125 \mathrm{~ms}$ & $\mathrm{PC} 1-\mathrm{PC} 2: 0.1 \mathrm{~ms}$ & $\mathrm{PC} 2$ - PC1: $124.9 \mathrm{~ms}$ \\
\hline
\end{tabular}




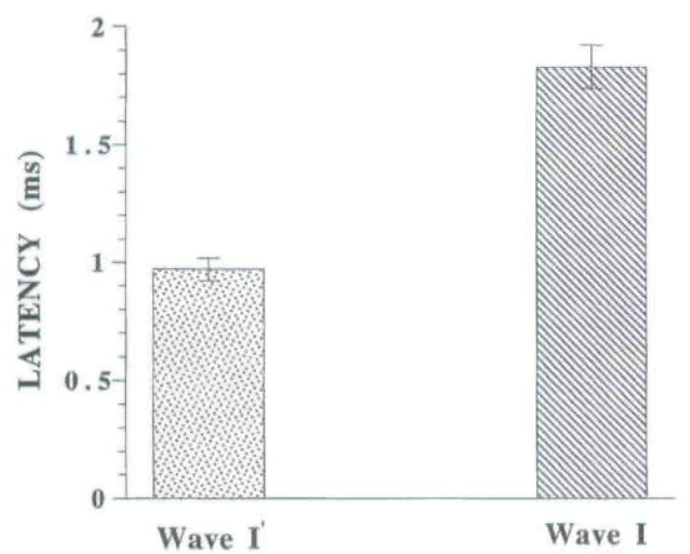

Fig. 2. Mean latency along with \pm 1 SD values of wave $\mathrm{I}^{\prime}$ and wave I recorded with standard-click stimuli.

has been established as the distal most portion of afferent VIIIth nerve fibers) and wave I' (whose anatomical generator is unknown but surmised to be the unmyelinated afferent VIIIth nerve dendrites) were analyzed and compared. Fig. 2 displays the mean latency for wave I' and wave I. The mean latency of wave I' was $0.97 \mathrm{~ms}$ (SD $0.10 \mathrm{~ms}$ ), which is consistent with previous data (Hughes \& Fino, 1980; Hughes et al., 1981; Benito et al., 1984; Moore \& Semela, 1989). Wave I mean latency was $1.83 \mathrm{~ms}$ (SD $0.10 \mathrm{~ms}$ ), which is also within normal limits. The latency of wave $\mathrm{I}^{\prime}$ exhibited a slightly larger variance than the latency of wave I.

The mean amplitudes for wave I' and wave I are depicted in Fig. 3. The mean amplitude recorded for $\mathrm{I}^{\prime}$ in the present investigation was $269.4 \mathrm{nV}$ (SD 133.1 nV), which fell slightly

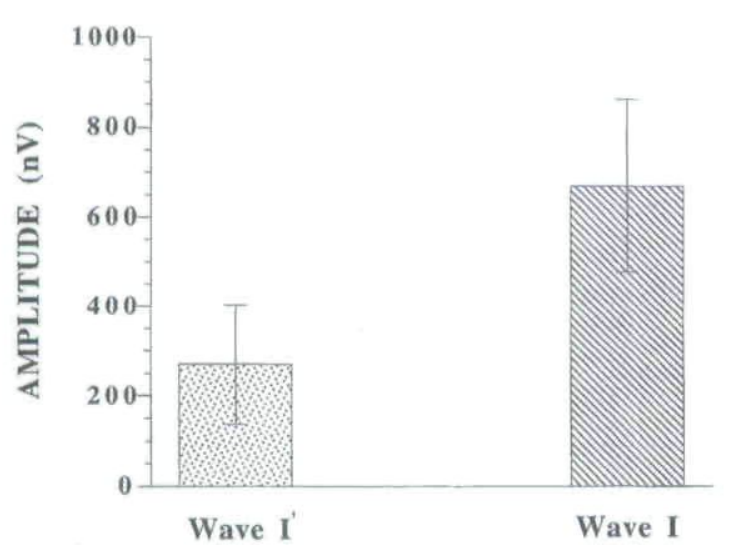

Fig. 3. Mean amplitude along with \pm 1 SD values of wave $\mathrm{I}^{\prime}$ and wave $\mathrm{I}$ recorded with standard-click stimuli.

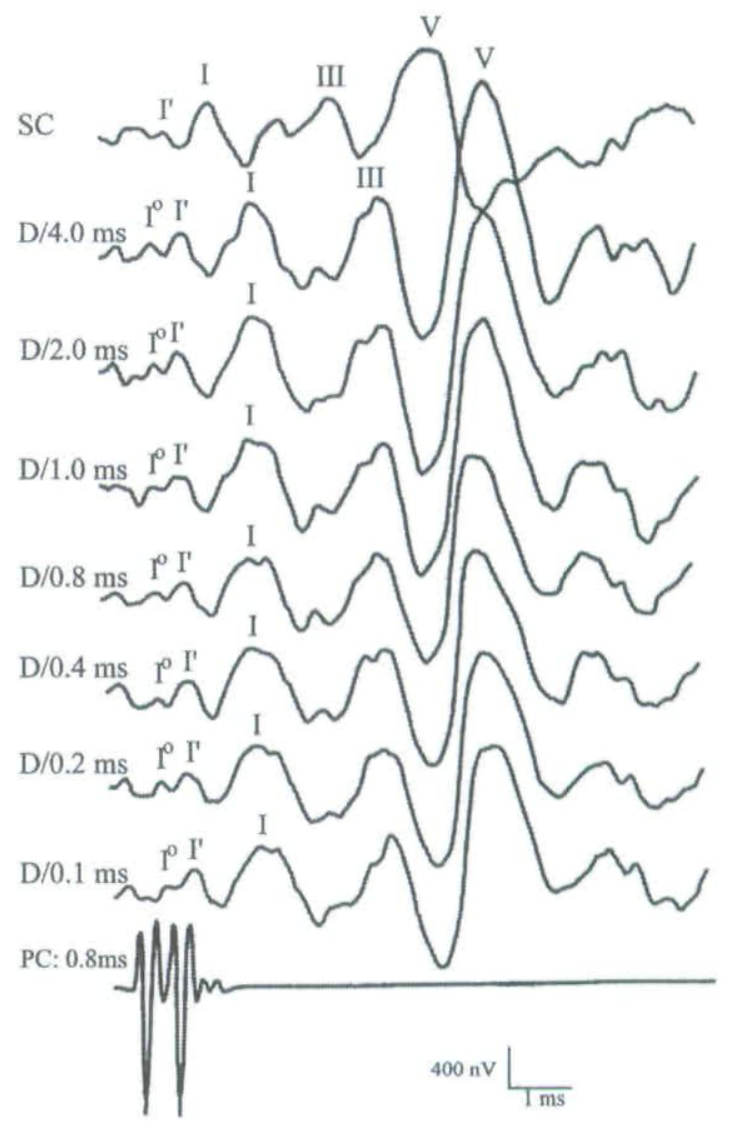

Fig. 4. Analog $\mathrm{ABR}$ waveform depicting wave $\mathrm{I}^{\circ}$, wave I' and waves I-V to standard click, followed by the derived ABR waveforms. The paired-click (PC) stimuli at $0.8 \mathrm{~ms}$ is depicted on the last panel.

below those measured by Moore et al. (1992), but are nearly consistent with amplitude values reported by Hughes \& Fino, 1980; Hughes et al., 1981). Mean amplitude value of wave I was $668.5 \mathrm{nV}$ (SD $192 \mathrm{nV}$ ). In order to examine the effectiveness of the paired-click paradigms to successfully differentiate between the SP and the EPSP, the derived responses were analyzed. Fig. 4 displays ABRs to a standard-click stimulus, and seven derived responses, along with the paired-click stimulus at $0.8 \mathrm{~ms}$. The seven derived ABRs increased in latency for all positive and negative peaks. The derived responses were further characterized by the appearance of two individual peaks, the first of which was referred to as $\mathrm{I}^{\circ}$; the second was designated as $\mathrm{I}^{\prime}$. There is an unexplained $1 \mathrm{~ms}$ delay in the derived responses that we attribute to the subtraction program, and is thus not a real latency shift, but a phase shift caused by the 


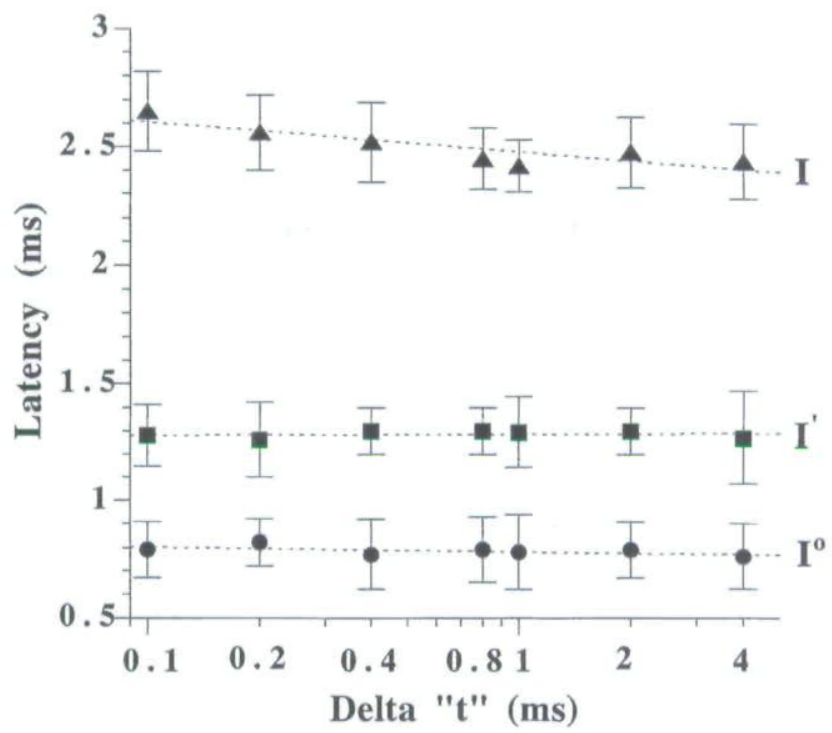

Fig. 5. Mean latency and $\pm 1 \mathrm{SD}$ deviation as a function of seven delta $t$ values for waves $\mathrm{I}^{\circ}, \mathrm{I}^{\prime}$ and $\mathrm{I}$. The regression line for each wave is also depicted.

algorithm of the subtraction program. Unfortunately, we were unable to address this problem due to an inability to obtain a source code from the manufacturer. The fact that later ABR waves did not shift in latency for the amount of the delta $t$ suggests that these latter waves were evoked by the first click.

Fig. 5 depicts the mean latency and \pm 1 SD for wave $\mathrm{I}^{\circ}$, wave $\mathrm{I}^{\prime}$ and wave $\mathrm{I}$, plotted as a function of each delta $t$. The mean latency for wave $\mathrm{I}^{\circ}$ ranged from $0.77 \mathrm{~ms}$ to $0.82 \mathrm{~ms}$ across delta $t$ values. Wave $\mathrm{I}^{\prime}$ mean latency ranged from $1.26 \mathrm{~ms}$ to $1.31 \mathrm{~ms}$ across the various delta $t$ values. Wave I mean latency ranged from 2.52 $\mathrm{ms}$ to $2.61 \mathrm{~ms}$. Variability was essentially similar across each delta $t$ value, but some

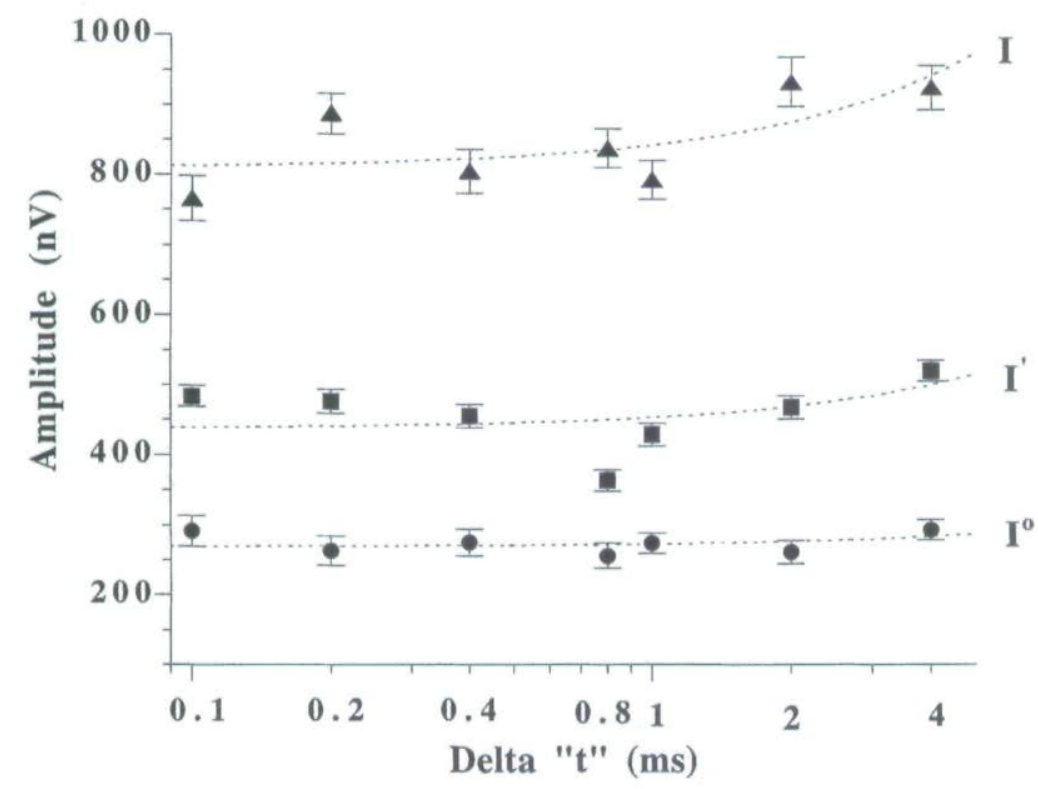

Fig. 6. Mean amplitude and $\pm 1 \mathrm{SD}$ as a function of seven delta $t$ values for waves $\mathrm{I}^{\circ}, \mathrm{I}^{\prime}$ and $\mathrm{I}$. The regression line for each wave is also depicted. 
a

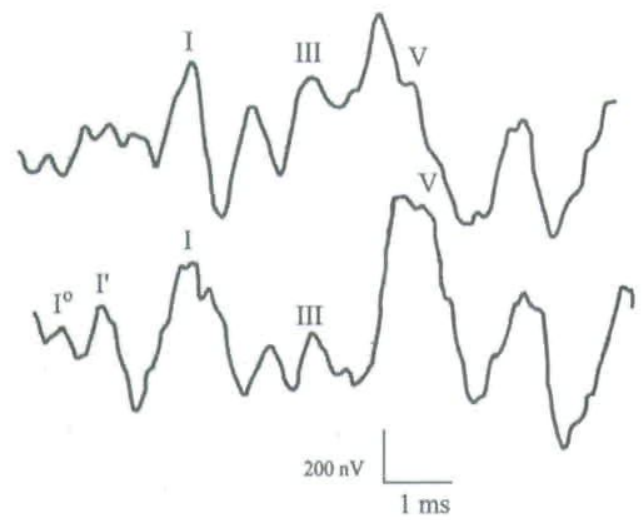

Fig. 7. (a) Analog ABR waveform obtained from regular paired-click stimuli. (b) Derived ABR waveform.

exceptions are noted. The identification of wave $\mathrm{I}^{\circ}$ was, at times, difficult to discern in the averaged traces for delta $t$ values greater than 0.8 ms.

Fig. 6 depicts the mean amplitudes and \pm 1 SD as a function of delta $t$ for waves $\mathrm{I}^{\circ}, \mathrm{I}^{\prime}$ and $\mathrm{I}$. The mean amplitude values for wave $\mathrm{I}^{\circ}$ ranged from $233.6 \mathrm{nV}$ to $302.0 \mathrm{nV}$. Mean amplitudes for wave I' ranged from $363.7 \mathrm{nV}$ to $520.3 \mathrm{nV}$. Mean amplitude for wave I ranged from $765.8 \mathrm{nV}$ to $934.0 \mathrm{nV}$.

Specific morphological changes were observed for various derived ABRs. They were characterized by the splitting of major and minor ABR peaks. It was not uncommon to observe that the positive portion of wave I to split into two and, in some instances, three smaller peaks. In some instances, wave III displayed bifid characteristics as well. The widening of all of the major and minor peaks (waves I-V) was also noted for all derived ABRs. In addition to these morphological characteristics, it was common to observe the disappearance and/or elimination of several minor peaks. For instance, the disappearance of wave II was not uncommon.

To appreciate the characteristics of the derived responses, $\mathrm{ABRs}$ were examined before and after the subtraction process. Fig. 7 illus- trates differences between the non-derived (a) and the derived (b) ABRs. In essence, the effects of both $\mathrm{PC} 1$ and $\mathrm{PC} 2$ are overlaid upon one another in Fig. 7a, making it impossible to identify specific peaks evoked by either click of the paired-click stimulus. The subtraction of the standard-click ABR from the paired-click ABR reflects differences, if any, between the two stimuli, namely PC2. Thus the derived response in Fig. $7 \mathrm{~b}$ is the result of the separation of the effects of PC2 from PC1 of the paired-click stimulus. This makes it possible to identify only those potentials evoked by PC2.

Table III summarizes the relationship (Kendall square test) between the latency of $\mathrm{I}^{\circ}, \mathrm{I}^{\prime}$ and wave I, as well as the amplitude of these same potentials. There was a low negative correlation of -0.30 for the latency of wave $I^{\circ}$. There was a positive but low correlation for the latency of wave $I^{\prime}(0.30)$, and a positive moderate correlation for wave I ( 0.70$)$. A low positive correlation for the amplitude of wave I (0.40) was obtained. There was a zero correlation for the amplitude of waves $\mathrm{I}^{\circ}$ and $\mathrm{I}^{\prime}$. Only the latency of wave I met our criteria of statistical significance at the 0.1 level.

\section{Discussion}

The paired-click ABRs were characterized by the appearance of two individual peaks, designated in this report as wave $\mathrm{I}^{\circ}$ and wave $\mathrm{I}^{\prime}$, both of which occurred before wave I. The latency values of each of these peaks $-\mathrm{I}^{\circ}, \mathrm{I}^{\prime}$, as well as I - were characterized by small standard deviations, suggesting minimal amounts of distribution from the mean for successive recordings from the three subjects. When comparing the range of latency and amplitude values, it became apparent that there was significantly more variability in the latter of the two responses. The amplitude of wave $\mathrm{I}^{\circ}$ was smaller than that of wave I' in each of the ABR waveforms. Changes in morphological characteristics, such as peak widening or splitting, were observed also

Table III. Correlational coefficients and significance values for the latency and amplitude of waves $I^{\circ}, I$ and $I$. Ampli $=$ Amplitude

\begin{tabular}{lllllll}
\hline Correlation test & Latency I $^{\circ}$ & Latency I $^{\prime}$ & Latency I & Ampli I $^{\circ}$ & Ampli I $^{\prime}$ & Ampli I \\
\hline Kendall & & & & & & \\
Significance & -0.30 & 0.30 & 0.70 & 0.0 & 0.0 & 0.40 \\
Coefficient & 0.217 & 0.217 & 0.012 & 0.440 & 0.440 & 0.088 \\
\hline
\end{tabular}


within all major and minor peaks of the derived ABRs. There was also an apparent shift in latency for all major and minor peaks of the derived responses.

In view of these data, several hypotheses were developed: (1) Wave $\mathrm{I}^{\circ}$ represents the cochlear $\mathrm{SP}$ response in the derived ABR; (2) wave $\mathrm{I}^{\prime}$ represents the summation of neural EPSPs in the derived ABRs; and (3) waves I through $\mathrm{V}$ remain in the derived ABRs because of the cumulative summation effects of PC1 in auditory nerve fibers, although phase shifts introduced by the subtraction process are apparent.

\section{Wave $I^{\circ}$ represents the cochlear summat- ing potential in derived ABRs}

In research using conditions similar to this study, SP amplitudes recorded from the external auditory meatus (EAM) of 48 normal ears stimulated with $116 \mathrm{~dB}$ peSPL clicks ranged from 0.82 to $0.01 \mu \mathrm{V}$ (mean 0.39; SD 0.17) (Coats, 1981). Eggermont (1976) used a $2000 \mathrm{~Hz}$ tone burst to elicit EAM-recorded SPs from 25 normal ears; these data revealed SP amplitude from $6.0 \mu \mathrm{V}$ to $0.36 \mu \mathrm{V}$ at $85 \mathrm{~dB} \mathrm{HL}$. SP amplitude was approximately 10 times larger than those measured from the EAM while using promontory methods of recording (Schmidt et al., 1974; Eggermont, 1976; Kumagami et al., 1982; Gibson et al., 1983). Gibson et al. (1983) recorded SP amplitude of 0.5 to $10 \mu \mathrm{V}$ (mean 3.90; SD 2.66) from 33 normal ears using clicks of $100 \mathrm{~dB}$ HL. These data suggest of course that electrode distance from the anatomical generator is an important factor for the size of the SP amplitude. This would account for the much smaller amplitude of $\mathrm{I}^{\circ}$ (SP) observed in this study since all data were obtained from surface electrodes.

Wave $\mathrm{I}^{\circ}$ revealed amplitudes that fell within and extended beyond the lower end of the SP voltages recorded from the EAM. Since one of the waveforms is involved in the derivation (subtraction) process, however, a direct comparison of the amplitude increase between the two responses is not possible (Henry \& Price, 1992). Therefore the smaller amplitudes seen in this study could be explained by the far-field method of recording used to collect the ABRs, as well as the lower stimulus intensity used.

Amplitude plotted as a function of delta $t$ shows very little fluctuation for wave $\mathrm{I}^{\circ}$. Continuing to reason in terms of wave $I^{\circ}$ representing a receptor potential, this observation becomes important. That is, the amplitude of a receptor potential is directly related to the amount of basilar membrane displacement, which is a direct result of the stimulus intensity. Thus, an increase in stimulus intensity would cause an increase in SP amplitude; or the opposite would be true of a decrease in stimulus intensity. Since intensity was kept at $105 \mathrm{~dB}$ peSPL for all ABR conditions, overt fluctuations in the amplitude of wave $\mathrm{I}^{\circ}$ amplitude, if it truly represents SP, should not have been observed.

The next logical step in the process to further support the hypothesis that wave $\mathrm{I}^{\circ}$ is the cochlear potential SP would be to compare the latency of this wave with that of SP latency. Unfortunately, current SP literature relative to specific measures of SP onset, peak and rise times is not readily available. However, most investigators generally document SP latency as $\leq 1 \mathrm{~ms}$ (Coats, 1981), and a visual inspection of several of the published reports reveals a similar trend. These data are consistent also with the mean latency documented in this study, with a range from $0.77 \mathrm{~ms}$ to $0.82 \mathrm{~ms}$.

Similar to amplitude, wave $\mathrm{I}^{\circ}$ latency plotted as a function of delta $t$ suggests that the latency of wave $\mathrm{I}^{\circ}$ does not exhibit any significant fluctuations. Given that the cochlear SP is a product of the activation of a specific group of hair cells and basilar membrane as determined by the frequency of the stimulus, successive stimulation of the hair cells by a stimulus that is unchanging, in terms of frequency, should reveal insignificant differences in successive latency measurements. In view of the fact that this study used a stimulus that remained constant, these data are considered promising. The latency characteristics of wave $\mathrm{I}^{\circ}$ are similar to those of a cochlear potential. Since the CM was cancelled and can be ruled out, the plausible alternative is that wave $\mathrm{I}^{\circ}$ from a surface electrode is the same as the SP of an electrode closer to the SP generator. That is, the $\mathrm{SP}$ is a presynaptic potential and is therefore not affected by adaptation, as adaptation and forward masking are closely related. In fact, the structure of the paired-click stimulus paradigm essentially represents a forward-masking paradigm. Given this information, adaptation effects caused by the paired-click stimulus paradigm are not expected for wave $\mathrm{I}^{\circ}$, especially if it truly reflects a SP.

In forward-masking paradigms, the amplitude of the AP to a click or tone presented after a 
masking tone (or click) increases as the time interval (ISI) between masker offset and click onset increases (McGill \& Rosenblith, 1951; Eggermont \& Spoor, 1973a, b; Eggermont \& Odenthal, 1974; Abbas, 1979; Harris \& Dallos, 1979; Abbas \& Gorga, 1981; Eggermont, 1985). Forward masking reveals also that response decrement is independent of the intensity level of the test tone, but is dependent on the level of the masking tone, and obviously dependent on delta $t$ (Smith, 1977). It has been reported also that frequency does not affect the decrement seen when the adapting tone level remains constant (Abbas \& Gorga, 1981). Furthermore, the longer the duration of the masking tone, the more adaptation effects are observed (Smith, 1977; Abbas \& Gorga, 1981; Eggermont, 1985).

Eggermont \& Spoor (1973a) summarized the effects of a forward-masking paradigm as being highly dependent on the ISI, stimulus intensity and stimulus duration. It was observed that AP amplitude decreased as ISI decreased and that an increased adapting stimulus level, as well as duration, was directly related to the amount of decrement. Forward-masking effects also revealed an increase in latency as the ISI decreased, and, as with amplitude, the increase in latency was directly related to increased stimulus intensity and duration. Furthermore, Eggermont \& Spoor (1973a) observed the distinct widening of $\mathrm{N}_{1}$ (of the AP complex, $N_{1} / P 1 / N 2$ ). It was also observed that peak $N_{1}$ widened as a function of a decrease of the ISI.

If wave $\mathrm{I}^{\circ}$ is representative of a neural potential, it would be expected that its amplitude would display a certain amount of decrement as the delta $t$ decreased. However, examination of Fig. 6 does not reveal any observable decline in amplitude as delta $t$ values increased. In the same vein, the manifestation of forward-masking effects within latency characteristics of a neural potential would include a latency decrement as delta $t$ increased. Examination of Fig. 5 does not reveal any overt changes in latency as a function of delta $t$. Thus, as a first approximation it would appear that wave $\mathrm{I}^{\circ}$ is not reflective of a neural potential.

Wave $I^{\prime}$ represents the summation of excitatory postsynaptic potentials in the derived $\boldsymbol{B A E P}$

Wave I has been associated with $\mathrm{N}_{1}$ of the AP complex, and its anatomical generator is thought to be the distal most portion of the VIIIth nerve. For this reason, amplitude and latency measures were compared for wave I and wave $\mathrm{I}^{\prime}$. This type of analysis should reveal similar functions if $\mathrm{I}^{\prime}$ truly represents a neural potential, namely, the EPSP. In contrast, differences between the functions of the two potentials would reveal just the opposite.

Inspection of the amplitude functions of all three waveforms reveals higher amounts of variability (as a function of delta $t$ ) for waves $\mathrm{I}^{\prime}$ and $I$, than for wave $I^{\circ}$. According to the forward-masking literature, the amplitude of a neural potential should increase as the delta $t$ increases. Examination of $\mathrm{I}^{\prime}$ amplitude as a function of delta $t$ shows that the results are consistent with these data. Furthermore, if $\mathrm{I}^{\prime}$ is a neural potential, its amplitude function should also mimic that of wave I, but not wave $I^{\circ}$. As expected, $\mathrm{I}^{\prime}$ amplitude increased as a function of an increase in delta $t$, whereas wave $\mathrm{I}^{\circ}$ amplitude remained fairly constant. It is therefore reasonable to surmise that wave $\mathrm{I}^{\prime}$ represents the summation of EPSPs in the derived ABR.

\section{Waves I through $V$ remain in the derived ABRs because of the summation of PC1 effects in auditory nerve fibers}

According to the basic premise of the pairedclick stimulus paradigm, PC2 should be delivered during either the absolute or relative refractory periods of the auditory nerve. This being true, PC2 should not evoke successive APs but should evoke EPSP activity. The calculation of the derived responses was done in order to separate the effects of $\mathrm{PC} 1$ from $\mathrm{PC} 2$, making it possible to record presumed EPSP in the human ABR. Additional effects, in the form of increased latency and amplitude, were expected in derived responses as well. However, the derived responses also contained peaks I through $\mathrm{V}$, but delayed by about $1 \mathrm{~ms}$. The appearance of these peaks in the derived responses indicated membrane potential fluctuations observed with AP propagation at the time $\mathrm{PC} 2$ was initiated. At 0.8 $\mathrm{ms}$, delta $t$ falls within the absolute refractory period and should not trigger APs in the VIIIth nerve. However, peaks I through V remain in the $0.8 \mathrm{~ms}$ derived response measurement. Since AP generation is not possible at this level, it is hypothesized that the residual effects of PC1 summate in auditory nerve fibers, and thus result in peaks $\mathrm{I}$ through $\mathrm{V}$ in the derived response 
(Abbas, 1979; Abbas \& Gorga, 1981). However, it may be that the most plausible explanation for the delay resides in a phase shift caused by the peculiarities of the subtraction algorithm and is not a true latency shift.

The fact that the majority of the correlation coefficients were not high (except wave I latency), nor reached statistical significance, is not surprising, albeit a few were in the positive direction ( $\mathrm{I}^{\prime}$ latency and $\mathrm{I}^{\prime}$ amplitude, wave I amplitude), one displayed a negative correlation $\left(\mathrm{I}^{\circ}\right.$ latency), and two showed no correlation $\left(\mathrm{I}^{\circ}\right.$ amplitude, I' amplitude). Only a limited number of subjects were tested, and those subjects that contributed to the data set were tested several times, thus showing very consistent data in the same direction. We designed the experiment in this manner since we wanted to ensure that each test run displayed reliable and readily recognizable waves of interest. It is thus surmised that if considerably more subjects had been added to the test pool, the results would have been more revealing.

To understand the morphological behavior of waves I through $\mathrm{V}$ in the derived responses, the forward-masking literature was, once again, consulted. Eggermont \& Spoor (1973a) were the first to describe $\mathrm{N}_{1}$ in both an unadapted and adapted condition with respect to morphology. They used a Gaussian distribution function to describe the shape of an unadapted $\mathrm{N}_{1}$. It was noted, however, that a decreasing ISI led to a deviation from the Gaussian distribution curve. A stimulus intensity of $50 \mathrm{~dB}$ SPL with an ISI of $4 \mathrm{~ms}$ displayed a broader $\mathrm{N}_{1}$, which was characterized with a 'bimodal' or double-peak shape. They explained this phenomenon as the result of $\mathrm{N}_{1}$ being the sum of two distributions. Each of the two individual distributions is Gaussian distributed and has approximately the same width, but different latencies and amplitudes. These data are consistent with the morphological changes referred to as 'peak widening' and 'peak splitting' in the derived responses of the present investigation.

\section{Acknowledgement}

We extend our sincere appreciation to Randy Robb for excellent technical assistance in computer programming. Supported in part by a grant from NSF.

\section{References}

Abbas PJ. Effects of stimulus frequency on adaptation in auditory nerve fibers. J Acoust Soc Am 1979; 65: 162-5.

Abbas PJ, Gorga MP. AP responses in forward-masking paradigms and their relationship to responses of auditory-nerve fibers. J Acoust Soc Am 1981; 69: 492-9.

Antoli-Candela F, Kiang NYS. Unit activity underlying the $\mathrm{N}_{1}$ potential. In: Naunton $\mathrm{R}$, Fernandez $\mathrm{C}$, editors. Evoked electrical activity in the auditory system. New York: Academic Press, 1978; 165-89.

Benito M, Falco P, Lauro A. Brain-stem auditory evoked potentials: description of the 'O' wave of possible electrocochlear origin. Electroencephalogr Clin Neurophysiol 1984; 58: 100.

Coats AC. The summating potential and Ménière's disease. Arch Otolaryngol 1981; 107: 199.

Dolan DF, Xi L, Nuttall AL. Characterization of an EPSPlike potential recorded remotely from the round window. J Acoust Soc Am 1989; 86: 2167-71.

Eggermont JJ, Spoor A. Cochlear adaptation in guinea pigs: a quantitative description. Audiology 1973a; 12: 193220.

Eggermont JJ, Spoor A. Masking of action potentials in the guinea pig cochlea, its relation to adaptation. Audiology 1973b; 12: 221-41.

Eggermont JJ, Odenthal DW. Electrophysiological investigation of the human cochlea: recruitment, masking and adaptation. Audiology 1974; 13: 1-22.

Eggermont JJ. Electrophysiological study of the normal and pathological human cochlea. I. Presynaptic potentials. Revue de Laryngologie, Otologie, Rhinologie 1976; 97 (Suppl): 487-95.

Eggermont JJ. Peripheral auditory adaptation and fatigue: a model oriented review. Hear Res 1985; 18: 57-71.

Furukawa T, Hayashida Y, Matsuura S. Quantal analysis of the size of excitatory post-synaptic potentials at synapses between hair cells and afferent nerve fibers in goldfish. J Physiol 1978; 276: 211-26.

Furukawa T. Sound reception and synaptic transmission in goldfish hair cells. Japan J Physiol 1986; 36: 1059-77.

Gibson WPR, Prasher DK, Kilkenny GPG. Diagnostic significance of transtympanic electrocochleography in Ménière's disease. Ann Otol-Rhinol-Laryngol 1983; 92: 155-9.

Harris DM, Dallos P. Forward masking of auditory nerve fiber responses. J Neurophysiol 1979; 42: 1083-107.

Henry KR, Price JM. Suprathreshold comparisons of derived and enhanced compound action potentials. Hear Res 1992; 63: 90-6.

Hughes J, Fino J. Usefulness of piezoelectric earphones in recording the brain stem auditory evoked potentials: a new early deflection. Electroencephalogr Clin Neurophysiol 1980; 48: 357-60.

Hughes J, Fino J. A review of generators of the brainstem auditory evoked potentials: contributions of an experimental study. Clin Neurophysiol 1985; 2 : 355-81.

Hughes J, Fino J, Gagnon L. The importance of phase of stimulus and the reference recording electrode in brain stem auditory evoked potentials. Electroencephalogr Clin Neurophysiol 1981; 50: 611-23.

Klinke R, Caird D, Löwenheim H, Moore E. Beeinflußt ein intracochleäres exzitatorisches postsynaptisches potential die Hirnstammpotentiale? Arch Oto-Rhino-Laryngol 1988(Suppl II): 159-60. 
Kumagami H, Nishida H, Baba M. Electrocochleographic study of Ménière's disease. Arch Otolaryngol 1982; 108: 284-8.

McGill WJ, Rosenblith WA. Electrical responses to two clicks: a simple statistical interpretation. Bull Math Biophys 1951; 13: 69-77.

Moore E, Semela JM. Relationship of electrophysiological hearing tests to rehabilitative audiology. $\mathrm{Br} \mathrm{J}$ Audiol 1989: 23: 39-40.

Moore EJ, Semela JM, Rakerd B, Robb R, Ananthanarayan AK. The I' potential of the brain-stem auditory-evoked potential. Scand Audiol 1992; 21: 153-6.

Ozdamar O, Dallos P. Synchronous responses of the primary auditory fibers to the onset of tone burst and

\section{BOOK REVIEW}

Hindley, P. and Kitson, N. (eds). Mental Health and Deafness. London: Whurr Publishers Ltd. 470 pp. 1999.

This first edition is an introductory text on mental health and deafness for care workers and mental health workers, i.e. those familiar with deaf people but not with mental health and those familiar with mental health but not with deaf people. In other words, the book is not intended as the complete knowledge in the field, but as a basic grounding and a good overview. Of 27 contributors, there is one otolaryngologist and one audiologist. Psychiatrists and psychologists constitute the main group of contributors.

In two main sections, it is schematic in structure, most of the chapters starting with the subheading 'Introduction' and ending with a conclusion or a summary. The first section, indicating a very thorough and broad approach, deals with assessment of different groups and is illustrated by the headings: Audiological assessment of people with special difficulties, child and adolescent psychiatry, adult psychiatry, deafness and intellectual impairment, psychological assessments, forensic psychiatry and deaf people, addictive behaviour and deafness.

I have some reservations, however, about the way audiology is treated. For example, in chapter 1, 'Deaf People in Society', in a passage concerning demographics of the deaf population, it is explained that severity of hearing impairment is expressed in terms of decibel (dB) loss, and that in the UK almost 20 of the population have hearing losses $>25 \mathrm{~dB}$. The fact that this is an average loss over the speech frequencies is their relation to compound action potentials. Brain Res 1978: 155: 169-75.

Palmer A, Russell I. Phase-locking in the cochlear nerve of the guinea-pig and its relation to the receptor potential of inner hair cells. Hear Res 1986; 24: 1-15.

Schmidt PH, Eggermont JJ, Odenthal DW. Study of Ménière's disease by electrocochleography. Acta OtoLaryngol 1974; 316 (Suppl): 75-84.

Siegel JH, Dallos P. Spike activity recorded from the organ of Corti. Hear Res 1986; 22: 245-8.

Smith RL. Short-term adaptation in single auditory nerve fibers: some poststimulatory effects. J Neurophysiol 1977; 40: 1098-112. first mentioned in chapter 11, dealing with acquired deafness. Furthermore, each chapter ends with a voluminous list of references, only very few of which are after 1995.

Section 2 comprises eight chapters dealing with management and intervention. The main focus is on psychodynamic therapies (psychotherapy and arts therapies) and behavioural and cognitive approaches. Other themes are family therapy in families with deaf members, interpreters in mental health settings, educational interventions, drug treatments and rehabilitation. The text gives a broad overview of theories and therapies but very few facts. One of the most important topics today-cochlear implant-is mentioned only in connection with cognitive behaviour therapy. The author states that 'the operation is controversial and viewed with suspicion by many within the Deaf community'. This was truth a few years ago, but attitudes to cochlear implant are changing. The topic is described in less than one page, and in my opinion is a gross underestimation of the importance of this new technology and its consequences. As in part one, each chapter ends with a voluminous list of references, only very few of which are after 1995.

In conclusion, the book might be an introductory text for those who are not familiar with mental health but have some knowledge of audiology and deaf people. Those familiar only with mental health will need some basic information on audiology. 